\title{
Taxa de gestação de receptoras de embriões bovinos tratadas com um análogo do GnRH no momento da inovulação
}

\author{
Pregnancy rate of bovine embryo recipients treated with a GnRH analogue at \\ the time of embryo transfer
}

LOIOLA, Marcus Vinícius Galvão ${ }^{1 *}$; PEREIRA, Danilo Francisco Campo'; VASCONCELOS, Livia Vieira ${ }^{1}$; LIMA, Marcos Chalhoub Coelho ${ }^{2}$; FERRAZ, Priscila Assis ${ }^{1}$; RODRIGUES, Alexandra Soares ${ }^{1}$; BITTENCOURT, Rodrigo Freitas ${ }^{2}$; JESUS, Edivânia Oliveira de ${ }^{3}$; RIBEIRO FILHO, Antonio de Lisboa ${ }^{2}$

\footnotetext{
${ }^{1}$ Universidade Federal da Bahia, Escola de Medicina Veterinária e Zootecnia, Programa de PósGraduação em Ciência animal nos Trópicos, Salvador, Bahia, Brasil.

${ }^{2}$ Universidade Federal da Bahia, Escola de Medicina Veterinária e Zootecnia, Departamento de Anatomia, Patologia e Clínicas, Salvador, Bahia, Brasil.

${ }^{3}$ Universidade Federal da Bahia, Escola de Medicina Veterinária e Zootecnia, Salvador, Bahia, Brasil.

*Endereço para correspondência: vinicius_mev@hotmail.com
}

\section{RESUMO}

O objetivo com este estudo foi avaliar a taxa de gestação de receptoras de embriões bovinos categorizadas de acordo com a qualidade do corpo lúteo (CL) e tratadas ou não com um análogo de GnRH (Fertirelina) no momento da inovulação. Somente os embriões viáveis foram selecionados para inovulação (1- excelente, 2bom e 3-regular) e transferidos. Para tanto, 272 novilhas foram distribuídas em seis grupos: CL1-S/GnRH (receptoras com área do $\mathrm{CL} \leq 1,5 \mathrm{~cm}^{2}$ e não tratadas com $\mathrm{GnRH}, \mathrm{n}=26$ ); CL1-C/GnRH (receptoras com área do $\mathrm{CL} \leq 1,5 \mathrm{~cm}^{2}$ e tratadas com GnRH, $\mathrm{n}=29$ ); CL2-S/GnRH (receptoras com área do CL entre 1,5 e $2,0 \mathrm{~cm}^{2}$ e não tratadas com $\mathrm{GnRH}, \mathrm{n}=46$ ); CL2-C/GnRH (receptoras com área do CL entre 1,5 e $2,0 \mathrm{~cm}^{2}$ e tratadas com $\mathrm{GnRH}, \mathrm{n}=45$ ); CL3$\mathrm{S} / \mathrm{GnRH}$ (receptoras com área do $\mathrm{CL}>2,0 \mathrm{~cm}^{2} \mathrm{e}$ não tratadas com GnRH, n=63); e CL3-C/GnRH (receptoras com área do $\mathrm{CL}>2 \mathrm{~cm}^{2}$ e tratadas com GnRH, n=63). As taxas de gestação das receptoras de embrião em relação à qualidade do CL e a aplicação ou não do GnRH foram respectivamente, 43,31 e $41,38 \%, 56,52$ e $46,67 \%$, e 46,03 e $42,86 \%$ para CL1-S/GnRH e CL1-C/GnRH, CL2-S/GnRH e CL2-C/GnRH, e CL3-S/GnRH e CL3-C/GnRH. Não houve diferença $(\mathrm{P}>0,05)$ entre as variáveis avaliadas. Portanto, a administração do análogo de GnRH (Fertirelina) não elevou a taxa de gestação em receptoras de embriões bovinos, independente da qualidade do CL, demonstrando que esta estratégia não foi eficaz em incrementar os índices de fertilidade.

Palavras-chave: corpo lúteo, fertirelina, transferência de embrião

\section{SUMMARY}

The aim of the study was to evaluate the pregnancy rate of bovine embryo recipients categorized according to quality of the corpus luteum (CL), treated or not with a GnRH analogue (Fertirelina) at the time of embryo transfer. Only viable embryos were selected for embryo transfer ( 1 - excellent, 2 - good, 3 - fair) and transferred. Therefore, 272 heifers were divided into six groups: CL1-S/GnRH (recipients with $\mathrm{CL}$ area $\leq 1,5 \mathrm{~cm}^{2}$ and not treated with GnRH, $\mathrm{n}=26$ ); CL1-C/GnRH (recipients with $\mathrm{CL}$ area $\leq 1,5 \mathrm{~cm}^{2}$ and treated with GnRH, n = 29); CL2-S/GnRH (recipients with $\mathrm{CL}$ area between 1,5 and $2,0 \mathrm{~cm}^{2}$ and not treated with GnRH, $\mathrm{n}=46$ ); CL2-C/GnRH (recipients with $\mathrm{CL}$ area between 1,5 and $2,0 \mathrm{~cm}^{2}$ and treated with GnRH, $\mathrm{n}=45$ ); CL3-S/GnRH (recipients witch CL area $>2 \mathrm{~cm}^{2}$ and not treated with GnRH, n = 63); and CL3-C/GnRH (recipients witch CL area $>2 \mathrm{~cm}^{2}$ 
and treated with GnRH, $\mathrm{n}=63$ ). Pregnancy rates of embryo recipients in relation to $\mathrm{CL}$ quality and the application or otherwise of GnRH were $43.31 \%$ and $41.38,56.52$ and $46.67 \%, 46.03$ and $42.86 \%$ for CL1-S/GnRH and CL1-C/GnRH, CL2-S/GnRH and CL2C/GnRH and CL3-S/GnRH and CL3-C/GnRH, respectively. Therefore, administration of GnRH analogue (Fertirelina) did not increase the pregnancy rate in bovine embryo recipients, independent of the quality of CL, demonstrating that this strategy is not effective in increasing fertility rates.

Keywords: corpus luteum, embryo transfer, fertirelina

\section{INTRODUÇÃO}

A transferência de embrião (TE) é uma importante biotecnologia para exploração do potencial reprodutivo dos rebanhos geneticamente superiores (VARAGO et al., 2008). Entretanto, diversos fatores influenciam a taxa de sobrevivência embrionária após a TE, dentre os quais se destaca a qualidade do corpo lúteo e consequentemente as concentrações plasmáticas de progesterona das receptoras (DISKIN \& MORRIS, 2008).

O estabelecimento da gestação envolve uma complexa interação entre 0 embrião, o ambiente uterino e o corpo lúteo (LIMA \& SOUZA, 2009). A progesterona provoca modificações no ambiente uterino, influenciando $\mathrm{O}$ crescimento e o desenvolvimento do concepto e consequentemente $\mathrm{o}$ bloqueio da luteólise (MANN et al., 2003).

Desta forma, há necessidade de estratégias hormonais, com o intuito de manter concentrações plasmáticas de progesterona elevadas para melhorar as sinalizações feto-maternal e o reconhecimento materno, visando $\mathrm{o}$ incremento nas taxas de gestação de receptoras de embriões (BÓ et al., 2012). Entre estas, destaca-se, o tratamento com análogos do Hormônio Liberador de Gonadotrofinas (GnRH), buscando modular a dinâmica folicular, melhorar a função do corpo lúteo original e possibilitar a formação de corpos lúteos acessórios (HOWARD et al., 2006; MACHADO et al., 2008; SILVA \& COSTA, 2005).

Vários pesquisadores ressaltaram a influência da qualidade do corpo lúteo no momento da inovulação, considerando que seria diretamente relacionada com a concentração plasmática de progesterona (LUTTGENAU et al., 2011) e que receptoras com corpos lúteos de menor tamanho apresentariam comprometimento nas taxas de gestação (VASCONCELOS et al., 2001).

Em uma pesquisa realizada por Baruselli et al. (2003) foi observado que a área do CL influenciou a concentração plasmática de progesterona e a taxa de concepção de receptoras de embrião Bos taurus taurus X Bos taurus indicus, onde CLs maiores proporcionaram maiores concentrações plasmáticas de progesterona e taxa de concepção.

Neste contexto a avaliação do corpo lúteo previamente ao tratamento com GnRH pode ser considerada um importante fator, principalmente, partindo do pressuposto que menores taxas de gestação são obtidas de receptoras com corpos lúteos menores, ou seja, de qualidade regular, podendo este tratamento ser mais eficaz nestes animais (SIQUEIRA et al., 2009).

Neste sentido, objetivou-se avaliar a taxa de gestação de receptoras de embriões bovinos categorizadas de acordo com a qualidade do corpo lúteo, tratadas ou não com um análogo de GnRH (Fertirelina) no momento da inovulação. 


\section{MATERIAL E MÉTODOS}

O experimento foi realizado em três fazendas, localizadas no centro-oeste baiano no período das chuvas, entre os meses de outubro de 2007 e abril de 2008, sendo realizada uma rodada de Transferência de Embrião por mês. Antes de iniciar o experimento as novilhas foram submetidos ao exame clínico-ginecológico e ultrassonografia transretal utilizando-se um transdutor linear de $6 \mathrm{MHz}$ (Pie-Medical, Falco 100, Maastricht, The Netherlands), verificando normalidade no trato reprodutivo e atividade cíclica regular. . Foram utilizadas como receptoras 272 novilhas mestiças (Bos taurus taurus $\mathrm{x}$ Bos taurus indicus) com idade de 2,5 $\pm 0,7$ anos, pesando $375,3 \pm 31,5 \mathrm{Kg}$, mantidas em piquetes de Brachiaria decumbens com sal mineral e água $a d$ libitum, sendo estas submetidas a um protocolo de transferência de embriões em tempo fixo (TETF) segundo Ribeiro Filho et al. (2011).

Os embriões utilizados foram oriundos de doadoras da raça Nelore, as quais passaram por um protocolo de superovulação de acordo com Ribeiro Filho et al. (2011) e foram inseminadas 12 e 24 horas após a indução das ovulações, utilizando-se sêmen criopreservado de fertilidade comprovada.

A recuperação dos embriões foi realizada sete dias após a inseminação artificial pelo método não cirúrgico por meio da lavagem do útero com DPBS (Embriocare $^{\circledR}$, Cultilab, Campinas, Brasil) aquecido a $37^{\circ} \mathrm{C}$. Após as colheitas os embriões foram transferidos para uma solução de manutenção e cultura contendo $0,4 \%$ de BSA (Embriocare Solução de Manutenção ${ }^{\circledR}$, Cultilab, Campinas, Brasil).
Posteriormente, foram classificados conforme seu estágio de desenvolvimento e qualidade. Somente aqueles com grau de desenvolvimento 4 (mórula), 5 (blastocisto inicial), 6 (blastocisto) e 7 (blastocisto expandido) e grau de qualidade 1 (excelente e bom), 2 (regular) e 3 (pobre), de acordo com a metodologia proposta por String Fellow e Seidel (1998), foram envasados em palhetas de $0,25 \mathrm{~mL}$ devidamente identificadas e transferidos.

No $17^{\circ}$ dia do protocolo de TETF pelo método não cirúrgico, os embriões envasados foram transferidos no corno uterino ipsilateral ao corpo lúteo. No momento da transferência, os ovários das receptoras foram examinados por ultrassonografia transretal para constatar a presença de corpo lúteo e classificá-los de acordo com sua área. Desta forma, neste momento, as receptoras foram categorizadas em animais com CL1 (área do $\mathrm{CL} \leq 1,5$ $\mathrm{cm}^{2}$ ), CL2 (área do CL entre 1,5 e 2,0 $\mathrm{cm}^{2}$ ) e CL3 (área do CL > 2,0 $\mathrm{cm}^{2}$ ) segundo metodologia utilizada por Baruselli et al. (2003).

As receptoras selecionadas foram distribuídas em seis grupos experimentais, em esquema fatorial $2 \times 3$, de acordo com a qualidade do corpo lúteo e o tratamento ou não com um análogo do GnRH: CL1-S/GnRH (receptoras com área do $\mathrm{CL} \leq 1,5 \mathrm{~cm}^{2}$ e não tratadas com GnRH, $\mathrm{n}=26$ ); CL1-C/GnRH (receptoras com área do $\mathrm{CL} \leq 1,5 \mathrm{~cm}^{2} \mathrm{e}$ tratadas com GnRH, n=29); CL2$\mathrm{S} / \mathrm{GnRH}$ (receptoras com área do $\mathrm{CL}$ entre 1,5 e $2,0 \mathrm{~cm}^{2}$ e não tratadas com GnRH, $\mathrm{n}=46$ ); $\mathrm{CL} 2-\mathrm{C} / \mathrm{GnRH}$ (receptoras com área do CL entre 1,5 e $2,0 \mathrm{~cm}^{2}$ e tratadas com GnRH, n=45); CL3-S/GnRH (receptoras com área do $\mathrm{CL}>2,0 \mathrm{~cm}^{2}$ e não tratadas com $\mathrm{GnRH}$, $\mathrm{n}=63$ ); e CL3-C/GnRH (receptoras com área do $\mathrm{CL}>2 \mathrm{~cm}^{2} \mathrm{e}$ tratadas com $\mathrm{GnRH}, \mathrm{n}=63$ ) 
Nos grupos tratados com GnRH foram administrados no momento da inovulação $100 \mu \mathrm{g}$ de um análogo do GnRH, Acetato de Fertirelina i.m. $\left(\right.$ Fertigen $^{\circledR}$, MSD Saúde Animal, São Paulo, Brasil) enquanto os grupos não tratados com este fármaco receberam $2 \mathrm{~mL}$ de solução salina a $0,9 \%$ i.m. Os embriões transferidos foram estratificados e equitativamente distribuídos nos diferentes grupos experimentais de forma homogênea em relação ao estágio de desenvolvimento e qualidade embrionária.

O diagnóstico de gestação foi realizado aos 30 dias após a inovulação com auxilio de ultrassom. Foi considerado como diagnóstico positivo de gestação a presença da vesícula embrionária $\mathrm{c}$ om um embrião viável (batimento cardíaco). A taxa de gestação foi calculada dividindo o total de receptoras gestantes pelo total de receptoras inovuladas.

Para as análises estatísticas das características avaliadas, foi empregado o pacote estatístico Statistical Analysis System (SAS INSTITUTE, 1996) com nível de significância de 0,05. Para se comparar a taxa de gestação utilizou-se um estudo de dispersão de frequências, através do PROC FREQ, empregandose o teste de qui-quadrado $\left(\chi^{2}\right)$. Na primeira fase das análises levou-se em consideração apenas a aplicação ou não do GnRH, em uma segunda etapa comparou-se ao mesmo tempo os tratamentos, qualidade do corpo lúteo e aplicação ou não do $\mathrm{GnRH}$ em relação as taxas de gestação das receptora. Para todas as avaliações levou-se em consideração o efeito do estágio de desenvolvimento embrionário, qualidade embrionária, local de realização do experimento e mês de realização do mesmo sobre a taxa de gestação.

A presente pesquisa recebeu aprovação do COMITÊ DE ÉTICA E BEM ESTAR ANIMAL, conforme O Protocolo n ${ }^{\circ}$ 27/2012.

\section{RESULTADOS E DISCUSSÃO}

Não houve efeito do estágio de desenvolvimento embrionário, da qualidade dos embriões inovulados, do local de realização do experimento e do mês de realização do mesmo sobre a taxa de gestação nos diferentes tratamentos. A taxa de gestação total das receptoras foi de 46,32\% (126/272). Levando-se em consideração apenas o tratamento ou não com GnRH no momento da inovulação, ou seja, sétimo dia do ciclo estral, a taxa de gestação do grupo tratado foi de 43,80\% (60/137) já o grupo dos animais não tratados alcançou uma taxa de 48,89\% (66/135). Não houve diferença $(\mathrm{P}>0,05)$ na taxa de gestação entre os grupos, resultados apresentados (Tabela 1).

Tabela 1. Taxa de gestação total de receptoras de embrião tratadas ou não com um análogo de GnRH

\begin{tabular}{lcc}
\hline \multirow{2}{*}{ Tratamentos } & \multirow{2}{*}{$\mathrm{N}^{\mathrm{o}}$ de animais } & Taxa de gestação \\
\cline { 3 - 3 } & 137 & $\mathrm{~N}^{\circ}(\%)$ \\
\hline $\mathrm{C} / \mathrm{GnRH}$ & 135 & $60(43,80)$ \\
S/GnRH & 272 & $66(48,89)$ \\
\hline Total & $126(46,32)$ \\
\hline Não houve diferença $(\mathrm{p}>0,05)$ entre os tratamentos. \\
C/GnRH - receptoras tratadas com GnRH; S/GnRH - receptoras não tratadas com GnRH.
\end{tabular}


Os achados deste experimento corroboram aos de Galimberti et al. (2001), os quais também não encontraram diferença entre as taxas de gestação do grupo de receptoras tratadas com $\mathrm{GnRH}$ no $11^{\circ}$ dia do ciclo estral e o grupo controle. Seguindo esta mesma linha de pesquisa, Fonseca et al. (2001) também não encontraram influência do tratamento com GnRH em receptoras ao tratá-las no quinto dia do ciclo estral. $\mathrm{O}$ que demonstra que a utilização deste fármaco em diferentes momentos do ciclo estral, não foi eficiente em aumentar a fertilidade das receptoras.

Estes resultados podem ser atribuídos à aplicação tardia do GnRH, não observando-se assim, o possível efeito deste fármaco sobre o desenvolvimento embrionário, uma vez que, o pico de síntese proteica que é necessário para o desencadeamento do mecanismo de reconhecimento materno possivelmente ocorre em estágios mais precoce do desenvolvimento embrionário (LONERGAN, 2011).

Contrastando com os resultados acima citados, Bó et al. (2012) verificaram que a aplicação do $\mathrm{GnRH}$ no sétimo dia do ciclo estral, semelhante ao que foi realizado no presente estudo, incrementou significativamente as taxas de gestação em receptoras de embriões bovinos (53,5\%, 53/99), porém segundo esses autores a taxa de gestação das receptoras do grupo controle foi menor que o esperado normalmente, $28,6 \%$ (28/98), o que pode ter contribuído para está diferença significativa.

Diversos estudos têm demonstrado uma correlação positiva e significativa entre a avaliação do corpo lúteo e a concentração plasmática de progesterona (VASCONCELOS et al., 2001; BARUSELLI et al., 2003; PUGLIESI et al., 2014). Além disso, receptoras com corpos lúteos de maior área apresentaram taxas de gestação significativamente superiores (ANDRADE et al., 2012). Assim, a qualidade do corpo lúteo poderia mascarar um impacto positivo do tratamento com GnRH sobre as taxas de gestação de receptoras de embriões bovinos e por isso os animais foram realocados de acordo com a qualidade do corpo lúteo.

Quando avaliado ao mesmo tempo o tratamento ou não das receptoras com GnRH e a qualidade do corpo lúteo, não verificou-se influência $(\mathrm{P}>0,05)$ deste fármaco sobre as taxas de gestação (Tabela 2).

Tabela 2. Taxa de gestação de receptoras de embrião categorizadas de acordo com a área do corpo lúteo e o tratamento ou não com um análogo do GnRH

\begin{tabular}{|c|c|c|c|}
\hline \multicolumn{2}{|c|}{ Tratamentos } & \multirow{2}{*}{$\mathrm{N}^{\mathrm{o}}$ de animais } & \multirow{2}{*}{$\begin{array}{c}\text { Taxa de gestação } \\
\mathrm{N}^{\mathbf{o}}(\%)\end{array}$} \\
\hline Área do CL & Aplicação de GnRH & & \\
\hline \multirow{2}{*}{ CL 1} & $\mathrm{~S} / \mathrm{GnRH}$ & 26 & $11(43,31)$ \\
\hline & $\mathrm{C} / \mathrm{GnRH}$ & 29 & $12(41,38)$ \\
\hline \multirow{2}{*}{ CL 2} & S/GnRH & 46 & $26(56,52)$ \\
\hline & $\mathrm{C} / \mathrm{GnRH}$ & 45 & $21(46,67)$ \\
\hline \multirow{2}{*}{ CL 3} & S/GnRH & 63 & $29(46,03)$ \\
\hline & $\mathrm{C} / \mathrm{GnRH}$ & 63 & $27(42,86)$ \\
\hline Total & & 272 & $126(46,32)$ \\
\hline
\end{tabular}

Não houve diferença $(\mathrm{p}>0,05)$ entre os tratamentos.

CL1 - receptoras com área do corpo lúteo $\leq 1,5 \mathrm{~cm}^{2}$; CL2 - receptoras com área do corpo lúteo entre 1,5 e $2,0 \mathrm{~cm}^{2}$; CL3 - receptoras com área do corpo lúteo $>2 \mathrm{~cm}^{2} ; \mathrm{S} / \mathrm{GnRH}$ - receptoras não tratadas com um análogo do GnRH; C/GnRH - Receptoras tratadas com um análogo do GnRH. 
A expectativa deste experimento era de que a administração de um análogo do GnRH fosse melhorar a taxa de gestação, principalmente, das receptoras de embriões que apresentavam corpos lúteos de menor área, considerados de pior qualidade, contudo, esta hipótese não se confirmou.

Uma justificativa para não se comprovar a hipótese acima citada poderia ser o fato da utilização do eCG (Gonadotrofina Coriônica Equina) no protocolo de sincronização das receptoras. Segundo Baruselli et al. (2012), este fármaco pode exercer um efeito luteotrófico favorecendo o reconhecimento materno da gestação destas fêmeas e podendo influenciar os índices de concepção, mantendo-os dentro dos padrões esperado de prenhez.

Outra possibilidade é que em rebanhos com fertilidade normal, desde que as concentrações de progesterona atinjam patamares limiares ou adequados, incrementos nas concentrações desse hormônio pode não ter efeitos significativos sobre a taxa de gestação (FONSECA et al., 2001). Os resultados mostraram que a administração do análogo de GnRH (Fertirelina) no momento da inovulação, da forma realizada neste experimento, não foi eficiente em elevar a taxa de gestação em receptoras de embriões bovinos, mesmo quando estas foram categorizadas de acordo com a qualidade do corpo lúteo, necessitando-se de mais estudos relacionando o tema.

\section{REFERÊNCIAS}

ANDRADE, G.A.; FERNANDES, M.A.; KNYCHALA, R.M.; PEREIRA JUNIOR, M.V.; OLIVEIRA, A.J.; NUNES, D.P.; BONATO, G.I.; SANTOS, R.M. Fatores que afetam a taxa de prenhez de receptoras de embriões bovinos produzidos in vitro. Revista Brasileira de Reprodução Animal, v.36, p.66-69, 2012.

BARUSELLI, P.S.; JACOMINI, J.O.; SALES, J.N.S.; CREPALDI, G. A importância do emprego da eCG em protocolos de sincronização para IA, TE e SOV em tempo fixo. Disponível em:

<www.sheepembryo.com.br/files/artig os/360.pdf>. Acesso em: 12 ago.

2012.

BARUSELLI, P.S.; MARQUES, M.O.; CARVALHO, N.A.T.; BERBER, R.C.A.; VALENTIM, R.; CARVALHO FILHO, A.F.; COSTA NETO, W.P. Dinâmica folicular e taxa de prenhez em novilhas receptoras de embrião (Bos taurus indicus $x$ Bos taurus taurus) tratadas com o protocolo "Ovsynch" para inovulação em tempo fixo. Brazilian Journal of Veterinary Research and Animal Science, v.40, p.96-106, 2003.

BÓ, G.A.; PERES, L.C.; CUTAIA, E.L.; PINCINATO, D.; BARUSELLI, P.S.; MAPLETOFT, R.J. Treatments for the synchronization of bovine recipients for fixed-time embryo transfer and improvement of pregnancy rates. Reproduction Fertility Development, v.24, p.272277, 2012.

DISKIN, M.G.; MORRIS, D.G. Embryonic and early foetal losses in cattle and other ruminants.

Reproduction in Domestic Animals, v.43, p.260-267, 2008.

FONSECA, J.F.; SILVA FILHO, J.M.; PALHARES, M.S.; RUAS, J.R.M.; PINTO NETO, A. Concentração plasmática de progesterona em novilhas 
Rev. Bras. Saúde Prod. Anim., Salvador, v.15, n.3, p.782-789 jul./set., 2014 http://www.rbspa.ufba.br ISSN 15199940

receptoras submetidas à administração rbST, GnRH ou hCG no quinto dia do ciclo estral. Arquivo Brasileiro de Medicina Veterinária e Zootecnia, v.53, p.451-458, 2001.

GALIMBERTI, A.M.; FONSECA, F.A.; ARAUJO, M.C.C.; COSTA, E.P.; FREITAS, C.; GUIMARÃES, J.D.; FERREIRA, A.M. Taxa de gestação e níveis plasmáticos de progesterona, em receptoras de embrião bovino, tratadas com buserelina após a inovulação.

Revista Brasileira de Zootecnia, v.30, p.353-359, 2001.

HOWARD, J.M.; MANZO, R.;

DALTON, J.C.; FRAGO, F.;

AHMADZADEH, A. Conception rates and serum progesterone concentration in dairy cattle administered gonadotropin releasing hormone 5 days after artificial insemination. Animal Reproduction Science, v.95, p.224233, 2006.

LIMA, I.M.T.; SOUZA A.L. Desenvolvimento e Sobrevivência de Embriões no Período de Préimplantação: Enfoque em Ruminante.

Revista Brasileira de Reprodução Animal, v.33, p.194-202, 2009.

LONERGAN, P. Influence of progesterone on oocyte quality and embryo development in cows.

Theriogenology, v.76, p.1594-1601, 2011.

LUTTGENAU, J.; ULBRICH, S.E.; BEINDORFF, N.; HONNENS, A.; HERZOG, K.; BOLLWEIN, H. Plasma progesterone concentrations in the midluteal phase are dependent on luteal size, but independent of luteal blood flow and gene expression in lactating dairy cows. Theriogenology, v.125, p.20-25, 2011
MACHADO, R.; BERGAMASCHI, M.A.; BARBOSA, R.T.; DE

OLIVEIRA, C.A.; BINELLI, M.

Ovarian fuction in Nelore (Bos taurus indicus) cows after post-ovulation hormonal treatments. Theriogenology, v.69, p.798-804, 2008.

MANN, G.E.; GREEN, M.P.; SINCLAIR, K.D.; DEMMERS, K.J.; FRAYC, M.D.; GUTIERRZ, C.; GARNSWORTHY, P.; WEBB, R. Effects of circulating progesterone and insulin on early embryo development in beef heifers. Animal Reproduction Science, v.79, p.71-79, 2003.

PUGLIESI, G.; OLIVEIRA, M.L.; SCOLARI, S.C.; LOPES, E.; PINAFFI, F.V.; MIAGAWA, B.T.; PAIVA, Y.N.; MAIO, J.R.G.; NOGUEIRA, G.P.; BINELLI, M. Corpus luteum development and fuction after supplementation of Long-Actiong Progesterone during the early luteal phase in beef cattle. Reproduction in Domestic Animals, v.49, p.85-91, 2014.

RIBEIRO FILHO, A.L.; RODRIGUES, A.S.; LIMA, M.C.C.; FERRAZ, P.A.; LOIOLA, M.V.G.; BITTENCOURT, R.F. Taxa de gestação de receptoras de embriões bovinos com diferentes graus de dificuldades no procedimento de inovulação. Ciência Animal

Brasileira, v.12, p.727-732, 2011.

SAS INSTITUTE. SAS user's guide for Windows Environment. Version 6.12. Cary, NC, 1996. 79p.

SILVA, J.C; COSTA, L.L. Luteotrophic influence of early bovine embryos and the relationship between plasma progesterone concentrations and embryo survival. Theriogenology, v.65, p.4960, 2005. 
Rev. Bras. Saúde Prod. Anim., Salvador, v.15, n.3, p.782-789 jul./set., 2014 http://www.rbspa.ufba.br ISSN 15199940

SIQUEIRA, L.G.B.; TORRES, C. A. A.; AMORIM, L. S.; SOUZA, E.D.; CAMARGO, L.S.A.; FERNANDES, C.A.C.; VIANA, J.H.M.

Interrelationships among morphology, echotexture, and function of the bovine corpus luteum during the estrous cycle. Animal Reproduction Science, v.115, p.18-28, 2009.

STRING FELLOW, D.A.; SEIDEL, S.M. Manual da Sociedade Internacional de Transferência de Embriões. Illinois: IETS, 1998. p.112113.
VARAGO, F.C.; MENDONÇA, L.F.; LAGARES, M.A. Produção in vitro de embriões bovinos: estado da arte e perspectiva de uma técnica em constante evolução. Revista Brasileira de Reprodução Animal, v.32, p.100109, 2008.

VASCONCELOS, J.L.M.; SARTORI, R.; OLIVEIRA, H.N.; GUENTHER, J.G.; WILTBANK, M.C. Reduction is size of the ovulatory follicle reduces subsequent luteal size and pregnancy rate. Theriogenology, v.56, p.307-314, 2001.

Data de recebimento: 15/10/2013

Data de aprovação: 23/09/2014 